\title{
ARTICLE
}

Clinical Study

\section{Galunisertib plus gemcitabine vs. gemcitabine for first-line treatment of patients with unresectable pancreatic cancer}

\author{
Davide Melisi ${ }^{1}$, Rocio Garcia-Carbonero ${ }^{2}$, Teresa Macarulla ${ }^{3}$, Denis Pezet ${ }^{4}$, Gael Deplanque ${ }^{5}$, Martin Fuchs ${ }^{6}$, Jorg Trojan ${ }^{7}$, \\ Helmut Oettle ${ }^{8}$, Mark Kozloff ${ }^{9}$, Ann Cleverly ${ }^{10}$, Claire Smith ${ }^{11}$, Shawn T. Estrem ${ }^{12}$, Ivelina Gueorguieva ${ }^{10}$, Michael M. F. Lahn ${ }^{13}$, \\ Al Blunt ${ }^{14}$, Karim A. Benhadji ${ }^{15}$ and Josep Tabernero ${ }^{16}$
}

BACKGROUND: Galunisertib is the first-in-class, first-in-human, oral small-molecule type I transforming growth factor-beta receptor (ALK5) serine/threonine kinase inhibitor to enter clinical development. The effect of galunisertib vs. placebo in patients with unresectable pancreatic cancer was determined.

METHODS: This was a two-part, multinational study: phase $1 \mathrm{~b}$ was a non-randomised, open-label, multicentre, and dose-escalation study; phase 2 was a randomised, placebo- and Bayesian-augmented controlled, double-blind study in patients with locally advanced or metastatic pancreatic adenocarcinoma considered candidates for first-line chemotherapy with gemcitabine. Patients were randomised 2:1 to galunisertib-gemcitabine $(N=104)$ or placebo-gemcitabine $(N=52)$. Gemcitabine dose was $1000 \mathrm{mg} / \mathrm{m}^{2}$ QW. Primary endpoints for phases $1 \mathrm{~b}$ and 2, respectively, were phase 2 dose and overall survival. Secondary objectives included tolerability and biomarkers.

RESULTS: Dose-escalation suggested a 300-mg/day dose. Primary objective was met: median survival times were 8.9 and 7.1 months for galunisertib and placebo, respectively (hazard ratio [HR] $=0.79$ [95\% credible interval: 0.59-1.09] and posterior probability $\mathrm{HR}<1=0.93$ ). Lower baseline biomarkers macrophage inflammatory protein-1-alpha and interferon-gamma-induced protein 10 were associated with galunisertib benefit.

CONCLUSIONS: Galunisertib-gemcitabine combination improved overall survival vs. gemcitabine in patients with unresectable pancreatic cancer, with minimal added toxicity. Future exploration of galunisertib in pancreatic cancer is ongoing in combination with durvalumab.

British Journal of Cancer (2018) 119:1208-1214; https://doi.org/10.1038/s41416-018-0246-z

\section{BACKGROUND}

Pancreatic cancer remains one of the most lethal and poorly understood human malignancies. ${ }^{1}$ It has the lowest 5-year relative survival rate among solid tumours at $8 \%^{2}$ and is projected to become the second leading cause of cancer-related death by 2030 in Western countries. ${ }^{3}$ Poor prognosis in pancreatic cancer is attributed to its early metastatic behaviour, aggressive clinical course, and limited efficacy of chemotherapeutic treatments. ${ }^{4,5}$

The transforming growth factor-beta (TGF- $\beta$ ) signalling pathway has one of the most complex and controversial roles in cancer. TGF- $\beta$ maintains homoeostasis in normal tissue; however, cancer cells have the capacity to circumvent this suppressive influence. Thus, pathological forms of TGF- $\beta$ signalling promote tumour growth, epithelial-to-mesenchymal transition, extracellular matrix remodelling, stemness and evasion of immune surveillance. ${ }^{6}$ Recent whole-genome or exome sequencing confirmed TGF- $\beta$ as the most recurrently mutated signal transduction pathway in pancreatic cancer. ${ }^{7}$

Inhibitors of TGF- $\beta$ signalling have been explored in pre-clinical models and showed enhanced anti-tumour activity in combination with gemcitabine. ${ }^{8}$ Galunisertib is the first orally bioavailable small-molecule inhibitor of the type I TGF- $\beta$ receptor (ALK5) serine/threonine kinase to enter clinical development. ${ }^{9}$

The present study was designed to determine an appropriate dose of galunisertib for combination with gemcitabine and to evaluate the combination for treatment of pancreatic cancer. Safety, pharmacokinetics, anti-tumour activity and biomarkers related to pancreatic cancer and TGF- $\beta$ signalling were also evaluated.

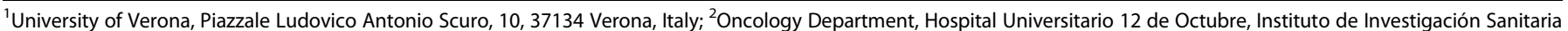

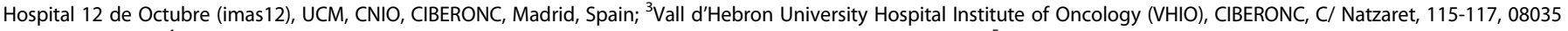

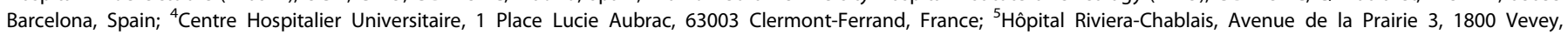

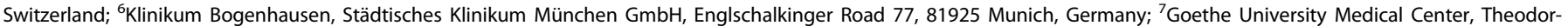

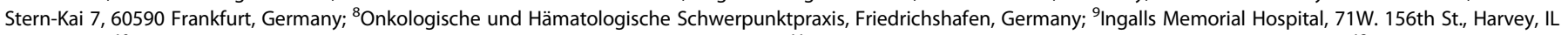

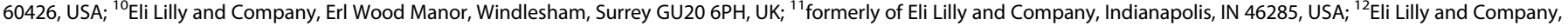

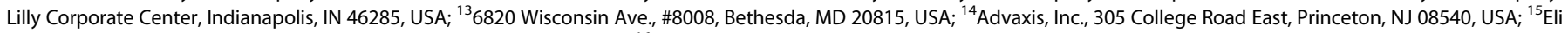

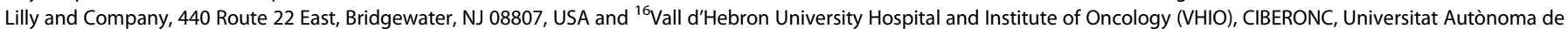
Barcelona, P. Vall d'Hebron 119-129, 08035 Barcelona, Spain

Correspondence: Davide Melisi (davide.melisi@univr.it)
}

Received: 21 May 2018 Revised: 14 July 2018 Accepted: 3 August 2018

Published online: 15 October 2018 


\section{METHODS}

Study design and participants

This was a multinational, two-part study of oral galunisertib in combination with gemcitabine. The first part (phase 1b) was a non-randomised, open-label, multicentre, dose-escalation phase in patients with solid malignancies who had not responded to anti-cancer therapies and who were eligible for gemcitabine therapy. The second part (phase 2) was a randomised, placeboand Bayesian-augmented controlled, double-blind study of galunisertib in combination with gemcitabine vs. gemcitabine plus placebo in patients with locally advanced or metastatic pancreatic adenocarcinoma at first presentation or after recurrence following radical surgery, and who were considered candidates for first-line chemotherapy with gemcitabine (Supplementary Figure S1). Previous adjuvant or neoadjuvant gemcitabine was allowed. Additional details in the Supplementary Materials.

\section{Randomisation and masking}

In phase 2, patients were randomly assigned 2:1 in the galunisertib group vs. the placebo group, using a dynamic randomisation procedure ${ }^{10}$ to minimise imbalance between treatment groups using the known prognostic factors of Eastern Cooperative Oncology Group (ECOG) performance (0 vs. 1 vs. 2), disease stage (stages II-III vs. IV), and previous gemcitabine treatment (adjuvant/neoadjuvant vs. no treatment), as well as investigational site.

\section{Procedures}

Patients were treated orally twice daily (morning and evening) with galunisertib at dose levels of 80,160 or $300 \mathrm{mg} /$ day during phase $1 \mathrm{~b}$ and $300 \mathrm{mg} /$ day or matching placebo during phase 2 for 14 days followed by 14 days off in a 28-day cycle. All patients (phase $1 \mathrm{~b}$ and 2 ) received gemcitabine via intravenous infusion at a dose of $1000 \mathrm{mg} / \mathrm{m}^{2}$ over $30 \mathrm{~min}$ ( $60 \mathrm{~min}$ maximum) once weekly for 7 weeks, followed by a week of rest from treatment. Given the selected centres, this regimen of gemcitabine was the most consensual at the time of study initiation. In order to characterise the galunisertib pharmacokinetic profile, the initial dose of gemcitabine was administered 7 days ( \pm 3 days) after the first dose of galunisertib or placebo was started. Subsequent gemcitabine cycles consisted of infusions once weekly for 3 of every 4 weeks. Additional details in the Supplementary Materials.

\section{Outcomes}

In phase $1 \mathrm{~b}$, the primary objective was to determine a safe/ tolerable phase 2 dose of galunisertib in combination with gemcitabine using a $3+3$ dose-escalation design. The phase 2 dose was expected to achieve a pre-defined plasma galunisertib exposure that was below levels associated with pre-clinical cardiovascular toxicity. ${ }^{11}$

In phase 2, the primary objective was to compare overall survival in the galunisertib and placebo groups using a Bayesian analysis. Key secondary objectives included evaluation of the pharmacokinetic profile and tolerability of galunisertib, comparison of the treatment groups with respect to progression-free survival and overall response rate as assessed by the investigators and central reviewers, evaluation of biomarker changes in tumour tissue and circulating blood, and assessment of patient-reported pain using the Brief Pain Inventory-short form (BPI-sf) and investigator-rated analgesic level.

\section{Statistical analysis}

Approximately 150 patients were to be randomly assigned and the final analysis/evaluation of overall survival was to be performed after approximately 135 events (deaths) had been recorded or 18 months after the last patient was enroled, whichever was sooner. The pre-specified Bayesian-augmented design consisted of borrowing data from trials in which patients with similar characteristics received the control treatment to optimise treatment comparison of interest and minimise the number of patients enroled in the concurrent control group. Using patient-level control data from two previous randomised gemcitabine trials ${ }^{12,13}$ and assuming a hazard ratio [HR] of 0.7 for survival, the study had $90 \%$ power to identify if the probability of $\mathrm{HR}<1$ was $>0.85$. The type 1 error rate was 0.16 .

Phase 2 efficacy analyses were conducted on the intention-totreat population, unless otherwise specified. This population included all patients randomly assigned to study treatment. The primary method of analysis for comparing overall survival between the treatment groups used a Bayesian exponentiallikelihood model with a hierarchical random-effects distribution on treatment effects. Additional details in the Supplementary Materials.

Sensitivity analyses included an additional Bayesian analysis with minimal borrowing of historical data for the control. In addition, to assess whether unexpected bias was introduced in this trial design, frequentist analyses of overall survival and progression-free survival using only the data from this study were conducted. Efficacy analyses of overall survival and progressionfree survival used Kaplan-Meier estimates and the log-rank test stratified by randomisation factors of Eastern Cooperative Oncology Group (ECOG) status, previous gemcitabine treatment, and disease stage (as assessed by investigator and confirmed by central re-read) (Supplementary Table S1). Cox proportional hazards models also adjusted for these randomisation factors, and stepwise analysis adjusted for multivariate factors including ECOG, previous gemcitabine treatment, baseline liver metastasis, sex, post-discontinuation systemic therapy and CA19-9. Overall response rates (complete response + partial response), clinical benefit rates (complete response + partial response + stable disease) and the exact $95 \%$ confidence interval $(\mathrm{Cl})$ were estimated for each group. The comparison between groups was done using the Fisher's exact test. All Bayesian analyses were carried out using the statistical software FACTS v2.4 (Berry Consultants LLC, Austin, TX) and all other analyses were carried out using SAS/Version 9.4.

Details on pharmacokinetics and exploratory analyses on circulating biomarkers are available in the Supplementary Materials.

\section{RESULTS}

This phase 1b/2 study was conducted between June 2011 and December 2016 at 24 centres in six countries. Of the 199 patients who entered screening for phase 2, 43 were screen-failures and 156 were randomised to study treatment, yielding 104 and 52 in the galunisertib and placebo intention-to-treat populations, respectively (Supplementary Figure S1). One patient assigned to galunisertib withdrew prior to receiving treatment, and 103 patients in the galunisertib group and 52 in the placebo group received at least one dose of study treatment (Supplementary Figure S2).

In phase 2, 87 (56\%) patients had an ECOG status of 1 and 151 (97\%) patients had stage III/IV disease at study entry (Supplementary Table S2). Within high-enroling centres, the dynamic randomisation successfully ensured balance between the treatments (data not shown). A median of 2.0 (range: 0-29) and 1.0 (range: 0-19) cycles were completed per patient for galunisertib and gemcitabine, respectively. Dose omissions occurred for galunisertib in 74 patients and for gemcitabine in 96 patients; dose reductions occurred for galunisertib in 14 patients and gemcitabine in 68 patients; dose discontinuations occurred for galunisertib for 34 patients and dose delays occurred for gemcitabine in 30 patients. A total of 84 patients died in the galunisertib group and 48 patients died in the placebo group. 

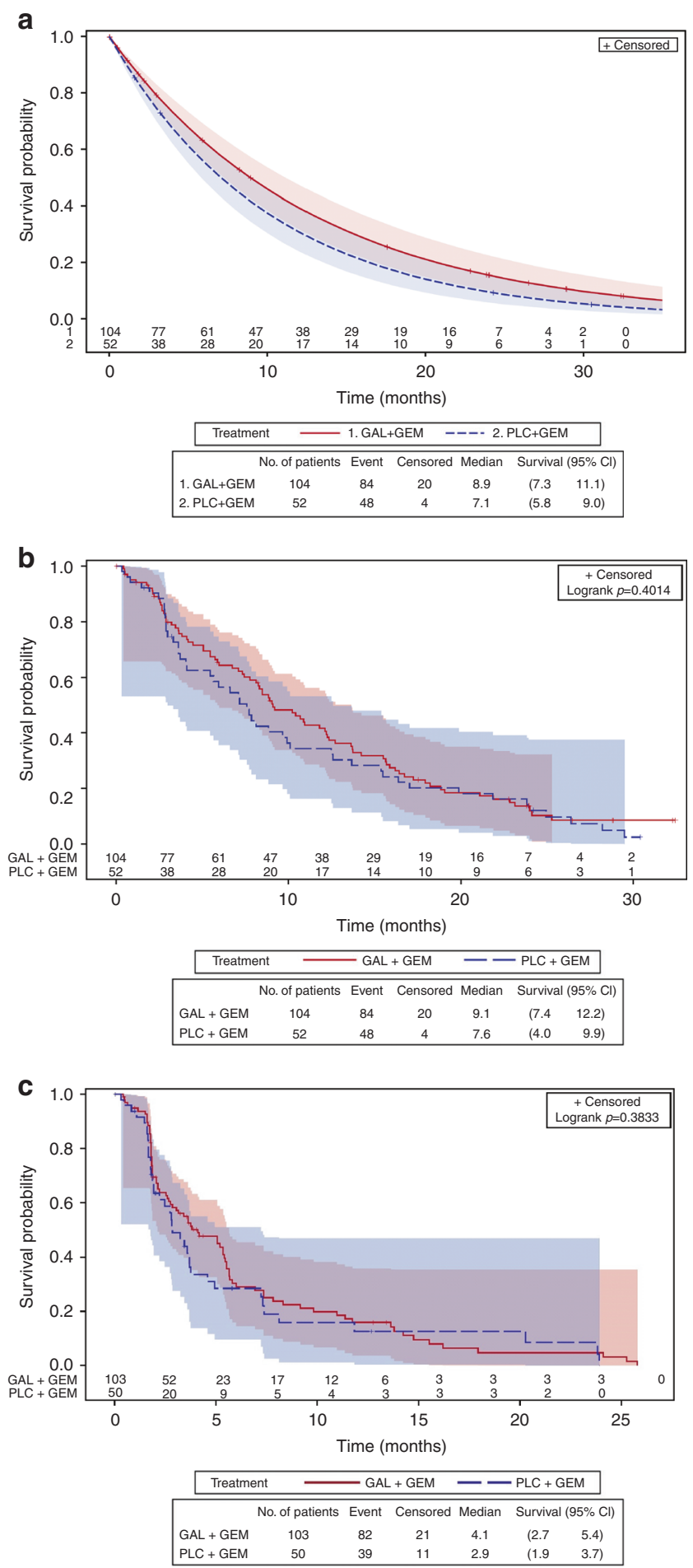

Fig. 1 Kaplan-Meier estimates of survival by Bayesian vs. frequentist analysis, with $95 \%$ Hall-Wellner confidence bands and numbers at risk. overall survival by a Bayesian analysis, b frequentist analysis and c progression-free survival by frequentist analysis. Data for one patient was missing from the progression-free survival analysis. $\mathrm{Cl}=$ confidence interval; $\mathrm{GAL}=$ galunisertib; $\mathrm{GEM}=$ gemcitabine; No.=number; PLC=placebo

From the primary Bayesian analysis, the median overall survival was 7.1 months (95\% credible interval [Crl]: 5.8-9.0) for the placebo group and 8.9 months (95\% Crl: 7.3-11.1) for the galunisertib group (Fig. 1a). The posterior median HR for overall survival was 0.79 (95\% Crl: $0.59-1.09)$ (Table 1). The posterior

probability that the $\mathrm{HR}$ is $<1$ was 0.93 , thus the primary objective was met.

The median overall survival for the control group from the sensitivity Bayesian analysis with minimal borrowing of historical data for the control was higher and closer to the results using only data from this study. The median overall survival was 7.4 months (95\% Crl: 5.7-10.0), the posterior median HR for overall survival was $0.83(95 \% \mathrm{Crl}: 0.59-1.19)$ (Table 1) and the posterior probability that the $\mathrm{HR}$ is $<1$ was 0.84 , just $<0.85$. Frequentist sensitivity analyses using a Cox regression model adjusted for prognostic factors estimated the HR between the two treatment groups as $0.86,95 \% \mathrm{Cl}$ : $0.60-1.23$ (Fig. 1 b, Table 1). No significant differences in progression-free survival were observed between the two treatments based on either investigator assessment or central readings (Fig. 1c, Supplementary Table S3). Previous treatment with gemcitabine was associated with improved overall survival ( $\mathrm{HR}=0.29,95 \% \mathrm{Cl}$ : 0.13-0.63; data not shown).

The overall response and clinical benefit rates as measured by central read assessment were higher for the galunisertib group, but the differences were not statistically significant (Supplementary Table S3). Results of pain analyses are in Supplementary Fig. S3.

Investigator-determined Common Terminology Criteria for Adverse Events $v 4.0$ grade $3-4$ events in phase 2 were mainly neutrophil count decreased, anaemia and fatigue (Table 2). In the galunisertib group vs. the placebo group, a higher percentage of patients had grade 3-4 neutrophil count decreased (36 [35\%] of 103 vs. 14 [27\%] of 52) and a slightly higher percentage of patients had grade $3-4$ fatigue (13 [13\%] of 103 vs. 5 [10\%] of 52 ); conversely, a lower percentage of patients had grade 3-4 anaemia (12 [12\%] of 103 vs. 9 [17\%] of 52) and grade $3-4$ platelet count decreased (8 [8\%] of 103 vs. 6 [12\%] of 52). There was a similar pattern for treatment-related grade 3-4 events (Supplementary Table S4). Few drug-related cardiac events were observed, and there was no significant difference in brain natriuretic peptide, cystatin $\mathrm{C}$ or ejection fraction between treatment groups. Thus, the cardiac safety was similar for both treatments. Likewise, no apparent difference in liver toxicity was observed (Supplementary Figure S4).

Galunisertib was rapidly absorbed into the systemic circulation, reaching maximum concentrations typically within $1 \mathrm{~h}$, and predicted exposures were within the therapeutic window (Supplementary Figure S5).

Baseline TGF- $\beta 1$ levels were balanced between treatment groups (Supplementary Table S2). Estimates of the HR generally favoured patients with lower TGF- $\beta 1$ at baseline (Supplementary Figure S6), suggesting that low TGF- $\beta 1$ at baseline is predictive of response to galunisertib treatment (HR of 0.84 in patients with low TGF- $\beta 1$ levels with an OS of 10.9 months for galunisertib group vs. 7.2 months for the placebo group). Patients in the galunisertib group with high TGF- $\beta 1$ at baseline had worse survival compared with the placebo (Supplementary Figure S7).

Assessment of the relationship between TGF- $\beta$ response and survival averaged across treatments (TGF- $\beta$ responders vs. nonresponders) gave a HR of $0.75(95 \% \mathrm{Cl}: 0.52-1.10)$ (data not shown). For TGF- $\beta$ responders, the median overall survival, pooled across treatments, was 10.1 months $(95 \% \mathrm{Cl}: 8.2-12.7)$ vs. 6.7 months (95\% Cl: $3.6-10.2)$ for TGF- $\beta$ non-responders. The percentage of patients achieving TGF- $\beta 1$ reduction $>20 \%$ was slightly higher in the galunisertib group (64 [69\%] of 93) compared with the placebo group (30 [60\%] of 50), with no significant difference in overall survival: 10.9 months $(95 \% \mathrm{Cl}$ : 8.2-13.7) and 9.8 months (95\% Cl: 7.2-15.5), respectively (Supplementary Figure S8).

After evaluating 279 plasma proteins from 156 patients, 249 proteins were evaluable for further examination. Of these, 31 proteins were identified as prognostic factors associated with survival $(p<0.001)$ across the study using the univariate Cox 


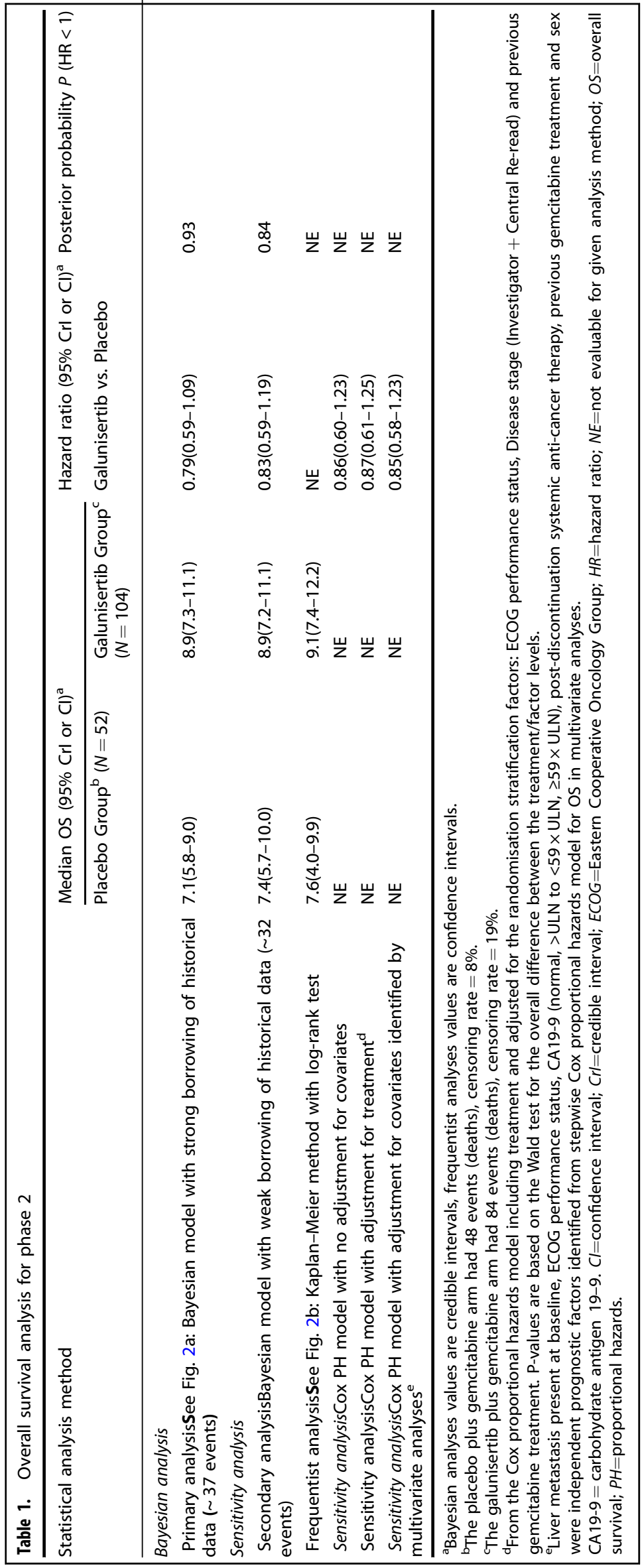


Galunisertib plus gemcitabine vs. gemcitabine for first-line treatment of...

D Melisi et al.

Table 2. Treatment-emergent adverse events ( $\geq 15 \%$ of any grade in phase $1 \mathrm{~b}$ or phase 2 in either group) by CTCAE term

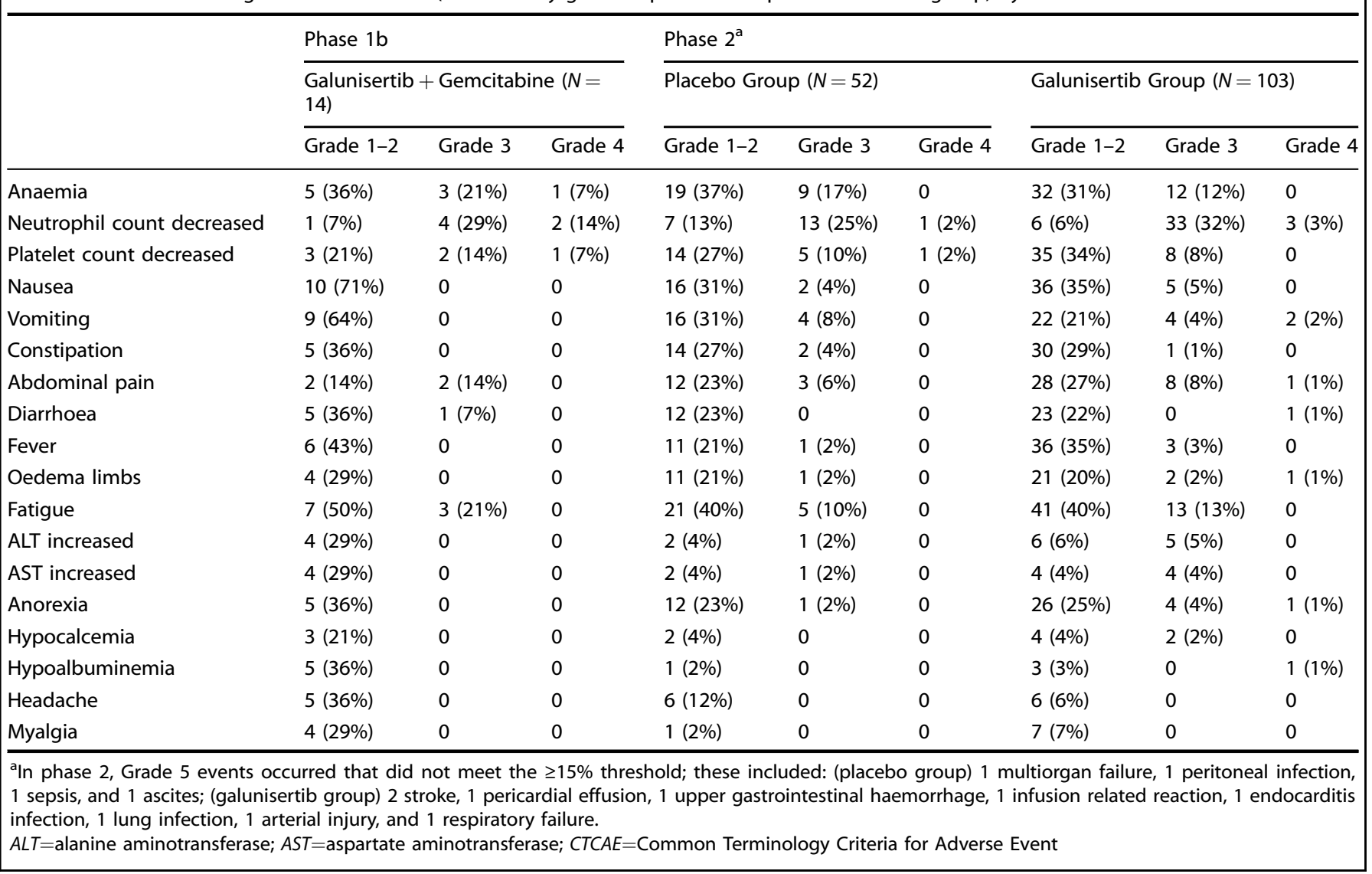

regressions model (Supplementary Figure S9). Additionally, 4 proteins were identified to be predictive factors associated with survival (unadjusted for other prognostic variables) following galunisertib treatment: interferon-gamma induced protein 10 (IP10) (>median at baseline $\mathrm{HR}=0.40,95 \% \mathrm{Cl}: 0.23-0.67$; $\leq$ median at baseline $\mathrm{HR}=1.35,95 \% \mathrm{Cl}$ : $0.83-2.21$ ), follicle-stimulating hormone (>median at baseline $\mathrm{HR}=1.40,95 \% \mathrm{Cl}$ : $0.83-2.37$; $\leq$ median at baseline $\mathrm{HR} 0.45,95 \% \mathrm{Cl}: 0.27-0.75)$, macrophage inflammatory protein-1 a (MIP-1a) (>median at baseline $\mathrm{HR}=0.38$, 95\% Cl: $0.22-0.66$; smedian at baseline $\mathrm{HR}=1.02,95 \% \mathrm{Cl}$ : 0.63-1.65) and plasminogen activator inhibitor 1 (>median at baseline $\mathrm{HR}=1.45,95 \% \mathrm{Cl}: 0.88-2.41$; $\leq$ median at baseline $\mathrm{HR}=$ $0.55,95 \% \mathrm{Cl}: 0.33-0.92)(p=0.01)$ (Fig. 2). The levels of these proteins did not change over time post-treatment in either group.

T-cell counts were reduced in both arms over the course of the study; however, patients who had $>50 \%$ decrease in T cells from baseline in the first three cycles of treatment had worse overall survival (Supplementary Figure S10).

\section{DISCUSSION}

The present study is the first randomised study of galunisertib in pancreatic cancer. We used a randomisation favouring the experimental group (2:1) and a pre-defined Bayesian-augmented design to enrich the control group data with pre-existing historical data. Using this approach, the novel combination of galunisertib plus gemcitabine showed an improvement in survival over gemcitabine alone. Considering the study design, the type I error of 0.16 may be considered high relative to other phase 2 studies; however, the primary analysis method exceeded the threshold probability $(p)$ required for the study to be positive quite substantially ( $p[\mathrm{HR}<1]=0.93$, whereas 0.85 was required to pass). Because this Bayesian-augmented design is rare in phase 2 studies, we performed sensitivity analyses to determine whether unexpected bias was introduced, including a frequentist analysis using only data from the study. In these supporting sensitivity analyses, the HR ranged from 0.83 to 0.87 , which still favours galunisertib treatment. Additionally, all known prognostic factors were well-balanced across treatment arms and therefore, minimal bias was introduced into the study by the 2:1 randomisation. None of the survival analyses were post hoc, but were all pre-specified in order to legitimately consider the totality of evidence from phase 2 study results when making decisions on future development. However, it should also be noted that the power of the study design was reduced when using the study data alone.

Over the past decades and at the time this study started, gemcitabine was considered the reference treatment in advanced pancreatic cancer. ${ }^{10}$ Only recently, the combination of gemcitabine with the new agent nab-paclitaxel ${ }^{14}$ or the three-drug combination regimen FOLFIRINOX ${ }^{11}$ were able to provide $a$ survival improvement over gemcitabine single agent. However, these regimens are significantly more toxic than gemcitabine and are recommended for patients who have an ECOG performance status $0-1,{ }^{12}$ whereas gemcitabine monotherapy remains recommended for $>40 \%$ of patients who have either an ECOG PS $2^{13}$ or a comorbidity profile that precludes more aggressive regimens. Therefore, there remains a clear unmet need for the development of gemcitabine-based regimens that are efficacious and more tolerable than combination chemotherapy options. Considering the overall safety profile of galunisertib plus gemcitabine did not significantly differ from gemcitabine alone, the present findings suggest that galunisertib plus gemcitabine may be an attractive alternative option, especially for frail patients who are unable to tolerate standard chemotherapy combination regimens. The 

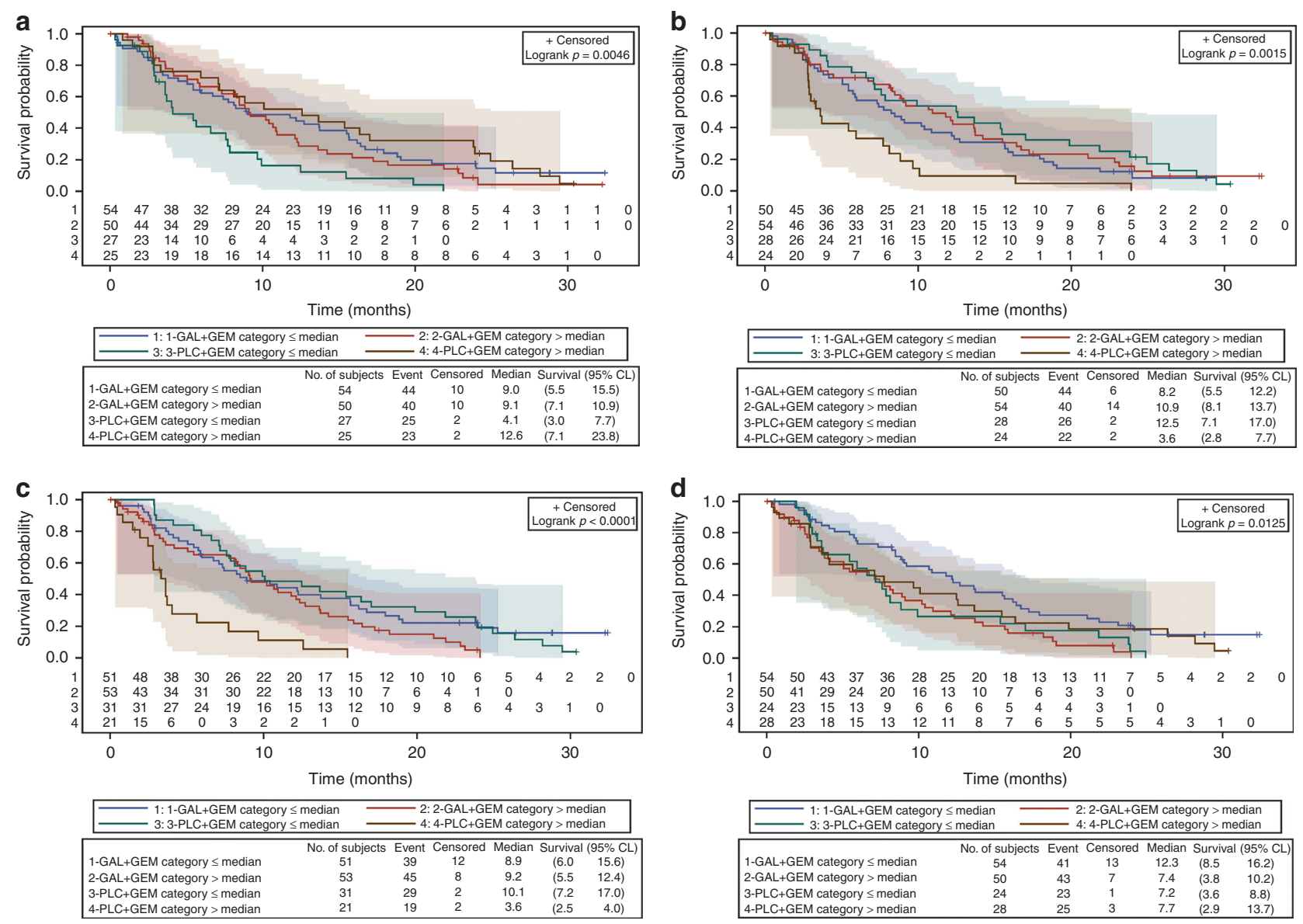

Fig. 2 Kaplan-Meier estimates of survival by potential predictive factor, with $95 \%$ Hall-Wellner confidence bands and numbers at risk. Overall survival by plasma level of a follicle-stimulating hormone, $\mathbf{b}$ interferon-gamma induced protein 10 , $\mathbf{c}$ macrophage inflammatory protein-1 alpha, and $\mathbf{d}$ plasminogen activator inhibitor $1 . C L=$ confidence limits; $G A L=$ galunisertib; $G E M=$ gemcitabine; No. $=$ number; $P L C=$ placebo

positive efficacy signals for galunisertib from this present study and its role in modulating the TGF- $\beta$ pathway have prompted interest in future validation of the efficacy of galunisertib plus gemcitabine as well as ongoing clinical trials of galunisertib with immunotherapeutic agents (anti-PD-L1 mAb durvalumab, NCT02734160). The intentions of these trials are to generate evidence for development of TGF- $\beta$ pathway inhibitors in combination with more modern therapeutic approaches.

Pancreatic cancer is characterised by a highly immunosuppressive tumour microenvironment that aids rather than controls cancer development and progression, ${ }^{15}$ and TGF- $\beta$ is commonly viewed as the most powerful immunosuppressive cytokine. ${ }^{16}$ In a recently integrated genomic analysis of pancreatic cancer, programmes enriched with a macrophage or T-cell co-inhibition specific signature co-segregated with poor survival. ${ }^{7}$ Additionally, we recently demonstrated that high plasma levels of cytokines involved in macrophage recruitment, including MIP-1a, or FOXP3 ${ }^{+}$ regulatory $T$ cells $\left(T_{\text {reg }}\right)$ enrichment, including IP-10, were negatively associated with pancreatic cancer patients' survival. ${ }^{17}$ Thus, we examined markers associated with inflammatory and other immunologic processes in pancreatic cancer to potentially select patients who may benefit from TGF- $\beta$ receptor inhibition. Indeed, we identified MIP-1a and IP-10 as two of the four most significant predictive factors for the efficacy of galunisertib plus gemcitabine. We confirmed that patients with high circulating levels of MIP-1a or IP-10 in the control group had a significantly shorter overall survival. Populations with such a poor prognosis received the greater benefit by the combination treatment with galunisertib. T-cell subsets were not different between the arms and patients with $<50 \%$ reduction in $T$ cells had a better overall survival than those with greater reductions, a relationship that may be linked to chemotherapy. Further studies addressing the effects of targeting TGF- $\beta$ signalling by galunisertib in macrophages and $\mathrm{T}_{\text {reg }}$ cells, and the consequent potential modulation of the immunosuppressive tumour microenvironment and chemoresistance in pancreatic cancer are warranted.

The pattern of treatment effect in the two TGF- $\beta$ responder groups was different. Within the responder group, median overall survival was similar across the treatments; however, median overall survival was improved for galunisertib plus gemcitabine in the non-responder group. Distribution of potential prognostic factors between the responder groups and treatment arms is under investigation, but this study was too small to ascertain possible reasons for the observation. Nonetheless, the association of reduced TGF- $\beta 1$ levels with improved survival has been observed in another disease, ${ }^{18}$ suggesting that reductions in TGF- $\beta 1$ levels could represent an additional tool to assess clinical benefit even if it may not be specific to a TGF- $\beta$ inhibitor.

In conclusion, the present study indicates a positive signal of efficacy for the combination of galunisertib and gemcitabine in improving overall survival vs. gemcitabine in patients with unresectable pancreatic cancer, with minimal added toxicity. Biomarker analyses provide evidence of patient subgroups with higher levels of cytokines recruiting macrophages or regulatory $\mathrm{T}$ cells that may benefit to a greater extent from treatment with galunisertib plus gemcitabine. Collectively, this evidence warrants further clinical development for galunisertib in combination with more modern chemotherapeutic or immunotherapeutic agents 
for patients with unresectable pancreatic cancer. One trial evaluating galunisertib in combination with durvalumab in pancreatic cancer is ongoing (NCT02734160).

\section{ACKNOWLEDGEMENTS}

The study team thanks patients and families for their willingness to participate in this study. Further, we thank all site staff and investigators at the institutions, the trial personnel at Eli Lilly and Company, and Covance. Durisala Desaiah, PhD, of Eli Lilly and Company and Larry Macke, ELS, Meghan Greenwood, PhD, Casie Polanco and Antonia Baldo of Syneos Health provided medical writing and editing support. Work in the unit of the corresponding author was supported by the Investigator Grant number 19111 through the Associazione Italiana per la Ricerca sul Cancro (AIRC).

\section{AUTHOR CONTRIBUTIONS}

The study was designed by D.M., M.M.F.L. and J.T. Data collection were conducted by D.M., R.G.-C., T.M., D.P., G.D., M.F., J.T., H.O., M.K., M.M.F.L., A.B. and J.T. All of the authors participated in data analysis and were involved in the drafting and editing of the manuscript, as well as the decision to publish. The funder provided the study drug, collaborated with investigators on the protocol, and was involved in the study design, data collection, analysis, interpretation and writing and preparation of this report.

\section{ADDITIONAL INFORMATION}

Supplementary Information is available for this paper at https://doi.org/10.1038/ s41416-018-0246-z.

Availability of data and materials: All data generated during the study is available at Eli Lilly.

PREVIOUS PRESENTATION: The data from this study were presented in part at ASCO 2013, AACR2016 and ASCO 2016.

Ethics approval and consent to participate: The study was conducted according to the principles of good clinical practice, applicable laws and regulations, the Council for International Organisations of Medical Sciences International Ethical Guidelines, and the Declaration of Helsinki. Each institution's ethical review board approved the study. All patients signed an informed consent document before study participation.

Competing interests: D.M. reports research funding from Shire, Incyte, and Celgene; a consulting role with Eli Lilly, Shire, Baxter and Incyte. G.D. reports research funding from Bavarian Nordici, Genentech, Janssen-Cilag, Roche, Sanofi, Eli Lilly, Exelixis, Sotio and Merck Serono; and travel fees from Roche, Amgen and Sanofi. M.F. reports a consulting role with Eli Lilly. J.T. reports a consulting role with Eli Lilly; speakers' bureau from Bayer, Amgen and Merck Serono; and travel fees from Bayer. M.K. reports honorarium from Eli Lilly, Genentech, and Roche; a consulting role with Eli Lilly, Genentech, and Roche; and speakers' bureau from Eli Lilly during the conduct of the study, Genentech, and Roche. A.C., C.S., S.T.E., I.G. and K.A.B. are all current employees and stockholders of Eli Lilly; M.M.F.L. is a former employee and stockholder of Eli Lilly and current employee of Incyte. A.B. is a current employee and stockholder of Advaxis, Inc. J.T. reports a consulting role with Eli Lilly, Amgen, Boehringer Ingelheim, Celgene, Chugai, Imclone, Merck, Merck Serono, Millenium, Novartis, Roche, Sanofi, Symphogen and Taiho. R.G.-C., T.M., D.P. and H.O. declare no competing interests.

\section{REFERENCES}

1. Melisi, D., Calvetti, L., Frizziero, M. \& Tortora, G. Pancreatic cancer: systemic combination therapies for a heterogeneous disease. Curr. Pharm. Des. 20, 6660-6669 (2014).

2. Siegel, R. L., Miller, K. D. \& Jemal, A. Cancer statistics, 2016. CA Cancer J. Clin. 66, 7-30 (2016)

3. Rahib, L. et al. Projecting cancer incidence and deaths to 2030: the unexpected burden of thyroid, liver, and pancreas cancers in the United States. Cancer Res. 74, 2913-2921 (2014)

4. Vaccaro, V. et al. Metastatic pancreatic cancer: is there a light at the end of the tunnel? World J. Gastroenterol. 21, 4788-4801 (2015).

5. Tamburrino, A., Piro, G., Carbone, C., Tortora, G. \& Melisi, D. Mechanisms of resistance to chemotherapeutic and anti-angiogenic drugs as novel targets for pancreatic cancer therapy. Front. Pharmacol. 4, 56 (2013).

6. Massagué, J. TGFbeta in cancer. Cell 134, 215-230 (2008).

7. Bailey, P. et al. Genomic analyses identify molecular subtypes of pancreatic cancer. Nature 531, 47-52 (2016).

8. Melisi, D. et al. LY2109761, a novel transforming growth factor-beta receptor type I and type II dual inhibitor, as a therapeutic approach to suppressing pancreatic cancer metastasis. Mol. Cancer Ther. 7, 829-840 (2008).

9. Herbertz, S. et al. Clinical development of galunisertib (LY2157299 monohydrate), a small-molecule inhibitor of transforming growth factor-beta signaling pathway. Drug Des. Devel Ther. 9, 4479-4499 (2015).

10. Burris, H. A. III et al. Improvements in survival and clinical benefit with gemcitabine as first-line therapy for patients with advanced pancreas cancer: a randomized trial. J. Clin. Oncol. 15, 2403-2413 (1997).

11. Conroy, T. et al. FOLFIRINOX versus gemcitabine for metastatic pancreatic cancer. N. Engl. J. Med. 364, 1817-1825 (2011).

12. Sohal, D. P. et al. Metastatic Pancreatic Cancer: American Society of Clinical Oncology Clinical Practice Guideline. J. Clin. Oncol. 34, 2784-2796 (2016).

13. Peixoto, R. D. et al. Eligibility of metastatic pancreatic cancer patients for first-line palliative intent nab-paclitaxel plus gemcitabine versus FOLFIRINOX. Am. J. Clin. Oncol. 40, 507-511 (2017).

14. Von Hoff, D. D. et al. Increased survival in pancreatic cancer with nab-paclitaxel plus gemcitabine. N. Engl. J. Med. 369, 1691-1703 (2013).

15. Sideras, K. et al. Role of the immune system in pancreatic cancer progression and immune modulating treatment strategies. Cancer Treat. Rev. 40, 513-522 (2014).

16. Travis, M. A. \& Sheppard, D. TGF- $\beta$ activation and function in immunity. Annu. Rev. Immunol. 32, 51-82 (2014).

17. Piro, G. et al. A circulating $\mathrm{TH} 2$ cytokines profile predicts survival in patients with resectable pancreatic adenocarcinoma. Oncoimmunology 6, e1322242 (2017).

18. Giannelli, G. et al. Evaluation of LY2157299 monohydrate (LY), a TGF-a receptor I kinase inhibitor in patients with advanced hepatocellular carcinoma: phase 2 study results of safety, efficacy and PK/PD. J. Hepatol. 60, S52-S53 (2014). (abstract 0126)

Open Access This article is licensed under a Creative Commons Attribution 4.0 International License, which permits use, sharing, adaptation, distribution and reproduction in any medium or format, as long as you give appropriate credit to the original author(s) and the source, provide a link to the Creative Commons license, and indicate if changes were made. The images or other third party material in this article are included in the article's Creative Commons license, unless indicated otherwise in a credit line to the material. If material is not included in the article's Creative Commons license and your intended use is not permitted by statutory regulation or exceeds the permitted use, you will need to obtain permission directly from the copyright holder. To view a copy of this license, visit http://creativecommons. org/licenses/by/4.0/.

(c) The Author(s) 2018 